\title{
Comparative Evaluation of Plasma Bile Acids, Dehydroepiandrosterone Sulfate, Hexadecanedioate, and Tetradecanedioate with Coproporphyrins I and III as Markers of OATP Inhibition in Healthy Subjects ${ }^{\text {\} }$
}

\author{
Hong Shen, Weiqi Chen, Dieter M. Drexler, Sandhya Mandlekar, Vinay K. Holenarsipur, \\ Eric E. Shields, Robert Langish, Kurex Sidik, Jinping Gan, W. Griffith Humphreys, Punit Marathe, \\ and Yurong Lai
}

Pharmaceutical Candidate Optimization (H.S., W.C., R.L., J.G., W.G.H., P.M., Y.L.) and Global Biometrics Sciences (K.S.), BristolMyers Squibb Company, Princeton, New Jersey; Pharmaceutical Candidate Optimization, Bristol-Myers Squibb Company, Wallingford, Connecticut (D.M.D., E.E.S.); Bristol-Myers Squibb India Pvt. Ltd. (S.M.) and Syngene International Ltd. (V.K.H.), Biocon BMS R\&D Center, Bangalore, India

Received February 17, 2017; accepted May 31, 2017

\begin{abstract}
Multiple endogenous compounds have been proposed as candidate biomarkers to monitor organic anion transporting polypeptide (OATP) function in preclinical species or humans. Previously, we demonstrated that coproporphyrins (CPs) I and III are appropriate clinical markers to evaluate OATP inhibition and recapitulate clinical drug-drug interactions (DDIs). In the present study, we investigated bile acids (BAs) dehydroepiandrosterone sulfate (DHEAS), hexadecanedioate (HDA), and tetradecanedioate (TDA) in plasma as endogenous probes for OATP inhibition and compared these candidate probes to CPs. All probes were determined in samples from a single study that examined their behavior and their association with rosuvastatin (RSV) pharmacokinetics after administration of an OATP inhibitor rifampin (RIF) in healthy subjects. Among endogenous probes examined, RIF significantly increased maximum plasma concentration $\left(C_{\max }\right)$ and area under the concentration-time
\end{abstract}

curve (AUC) $)_{(0-24 h)}$ of fatty acids HDA and TDA by 2.2- to 3.2-fold. For the 13 bile acids in plasma examined, no statistically significant changes were detected between treatments. Changes in plasma DHEAS did not correlate with OATP1B inhibition by RIF. On the basis of the magnitude of effects for the endogenous compounds that demonstrated significant changes from baseline over interindividual variations, the overall rank order for the AUC change was found to be $\mathrm{CP}$ I > CP III > HDA $\approx$ TDA $\approx$ RSV > > BAs. Collectively, these results reconfirmed that CPs are novel biomarkers suitable for clinical use. In addition, HDA and TDA are useful for OATP functional assessment. Since these endogenous markers can be monitored in conjunction with pharmacokinetics analysis, the CPs and fatty acid dicarboxylates, either alone or in combination, offer promise of earlier diagnosis and risk stratification for OATPmediated DDIs.

\section{Introduction}

The current paradigm for the evaluation of the in vivo inhibition potential of a new chemical entity (NCE) for human OATP1B1 and OATP1B3 activities is through administration of exogenous substrate drugs such as rosuvastatin (RSV) and atorvastatin. These drug-drug interaction (DDI) studies confer some risk to participants (Leung et al., 2012) and consume considerable resources and cost. Thus, development of a sensitive, specific endogenous biomarker to monitor OATP1B

This study was supported by Bristol-Myers Squibb Company. https://doi.org/10.1124/dmd.117.075531.

SThis article has supplemental material available at dmd.aspetjournals.org. activity and assess DDI liability in vivo would be very beneficial to early drug-development efforts. Use of endogenous biomarkers would obviate the need to administer a substrate drug and facilitate cost reduction as well as minimize DDI studies done in response to false positive predictions made using in vitro inhibition data. Moreover, the OATP inhibition liability of an investigational drug could be determined as early as first-in-human phase I clinical trials through monitoring endogenous biomarkers in conjunction with NCE pharmacokinetics analysis. Early organic anion transporting polypeptide (OATP) inhibition information would allow prioritization of clinical studies and optimization of phases II and III protocols by setting appropriate exclusion criteria for coadministered medicines. However, limited clinical investigations have been performed to

ABBREVIATIONS: AUC, area under the concentration-time curve; CA, cholic acid; CDCA, chenodeoxycholic acid; CP, coproporphyrin; CsA, cyclosporin A; DCA, deoxycholic acid; DDI, drug-drug interaction; DHEAS, dehydroepiandrosterone sulfate; GCA, glycocholic acid; GCDCA, glycochenodeoxycholic acid; GDCA, glycodeoxycholic acid; GUDCA, glycoursodeoxycholic acid; HDA, hexadecanedioate; HEK, human embryonic kidney; $I_{50}$, concentration required to inhibit transport by 50\%; LC-MS/MS, liquid chromatography-tandem mass spectrometry; LCA, lithocholic acid; NCE, new chemical entity; NTCP, sodium taurocholate cotransporting polypeptide; OAT, organic anion transporter; OATP, organic anion transporting polypeptide; OCT, organic cation transporter; RIF, rifampicin; RSV, rosuvastatin; TCA, taurocholic acid; TCDCA, taurochenode-oxycholic acid; TDA, tetradecanedioate; TDCA, taurodeoxycholic acid; THDCA/TUDCA, taurohyodeoxycholic acid/tauroursodeoxycholic acid; UDCA/HDCA, ursodeoxycholic acid/hyodeoxycholic acid. 
validate endogenous compounds as biomarkers of OATP1B1 and OATP1B3.

The OATP1B subfamily is involved in the transport of a wide range of substrates, including many endogenous substances. For example, coproporphyrins (CPs) I and III, byproducts of heme synthesis, are substrates for cynomolgus monkey and human OATP1B1 and OATP1B3 in vitro, as established using transporter-overexpressing cell lines (Bednarczyk and Boiselle, 2016; Shen et al., 2016). OATP transporters also transport bile acids and bilirubin. Bile acids (BAs), synthesized from cholesterol, are a family of amphipathic acidic steroids and play an important role not only in fat digestion and intestinal absorption of hydrophobic compounds but also regulate various functions via alteration of protein expression through binding to nuclear and membrane receptors (Marin et al., 2015). Once synthesized by the hepatocytes, BAs are efficiently secreted into the bile and then absorbed from the intestine. BAs in the portal blood are avidly taken up by the hepatocytes and are resecreted, setting up a continuous cycle between the intestine and the liver, a process known as enterohepatic circulation (Kullak-Ublick et al., 2004; Klaassen and Aleksunes, 2010). Sodium taurocholate cotransporting polypeptide (NTCP) mediates the hepatic uptake of various BAs by $\mathrm{Na}^{+}$-dependent mechanisms, whereas OATP1B1 and OATP1B3 mediate the uptake of primary as well as secondary bile acids, including taurocholic acid, chenodeoxycholic acid, and taurodeoxycholic acid, by $\mathrm{Na}^{+}$-independent mechanisms (Cui et al., 2001; Briz et al., 2006; Maeda et al., 2006, Yamaguchi et al., 2006; Neyt et al., 2016). Chu and colleagues (2015) demonstrated a trend of weak increases in the plasma exposures of glycocholic acid, glycochenodeoxycholic acid, and glycodeoxycholic acid caused by rifampin (RIF) in monkeys. Watanabe et al. (2015) found that dehydroepiandrosterone sulfate (DHEAS), a metabolite of steroid hormone dehydroepiandrosterone, is taken up into both human and monkey hepatocytes in a temperature-dependent and RIF-sensitive manner. DHEAS was increased in plasma followed by RIF treatment in cynomolgus monkeys. Although the magnitude of the fold-change in AUC of DHEAS was less than that of the statins used in the same monkey DDI study (1.1-fold versus 2.3- to 3.4-fold and 1.9-fold versus 10.2- to 13.8 -fold at 2 and $10 \mathrm{mg} / \mathrm{kg}$ RIF, respectively), the changes in DHEAS concentration were expected to be higher in humans than in monkeys on the basis of the results of in vitro hepatic uptake of DHEAS (Watanabe et al., 2015). More recently, using data available from genome-wide association studies and clinical samples from a DDI study between pravastatin and cyclosporin A (CsA), Yee et al. (2016) have identified 12 metabolites, including hexadecanedioate (HDA) and tetradecanedioate (TDA), that were significantly associated with reduced-function OATP1B1 genetic variants and exhibited elevated plasma concentrations after administration of CsA. In vitro experiments further confirmed HDA and TDA were substrates for OATP1B1 as well as organic anion transporter (OAT) 1 and OAT3. However, the plasma metabolite levels were analyzed at a single time point of 30 minutes after administration of CsA and pravastatin or pravastatin alone (Yee et al., 2016). Therefore, additional clinical studies are needed to better establish the sensitivity and the time course of the change from basal levels of these probes in the presence of an OATP inhibitor.

Since multiple endogenous molecules hold promise to be sensitive and specific markers of OATP activity in both preclinical species and humans, there is great interest in comparative assessments of the endogenous probes within a single human OATP DDI study. Thus, we analyzed the plasma levels of BAs, DHEAS, HDA, and TDA collected from a clinical DDI study between RIF and RSV using liquid chromatography-tandem mass spectrometry (LC-MS/MS) and compared these values to $\mathrm{CP}$ changes reported previously (Lai et al., 2016). In addition to OATP transport, further experiments were conducted in human embryonic kidney (HEK) cells expressing major renal drug transporters, including OATs 1-4, organic cation transporter (OCT) 2, multidrug and toxin extrusion protein (MATE) 1, and kidney-specific multidrug and toxin extrusion protein (MATE2K) for CPs I and III uptake to better define the transporter specificity of the probes. To evaluate whether there is substrate-dependent difference in the inhibition, a parallel set of $\mathrm{IC}_{50}$ values were determined against the transport of CPs I and III by OATP1B1, OATP1B3, and OATP2B1 in comparison with those against RSV.

\section{Materials and Methods}

Chemicals and Reagents. Dehydrocholic acid was purchased from MP Biomedicals (Solon, $\mathrm{OH}$ ). Dehydroepiandrosterone 3-sulfate and dehydroepiandrosterone 3 -sulfate- $\mathrm{d}_{5}$ were purchased from Cerilliant (Round Rock, TX). Hexadecanedioic acid- $\mathrm{d}_{28}$ and tetradecanedioic acid- $\mathrm{d}_{24}$ were obtained from CDN Isotopes (PointeClaire, Quebec, Canada). Rosuvastatin, probenecid, pyrimethamine, quinidine, rifampicin, rosuvastatin- $\mathrm{d}_{6}$, and coproporphyrin $\mathrm{I}^{15}{ }^{15} \mathrm{~N}_{4}(>97 \%$ purity) were purchased from Toronto Research Chemicals (North York, ON, Canada). Coproporphyrins I and III dihydrochloride ( $\geq 97 \%$ purity) were purchased from Frontier Scientific (Logan, UT). [ $\left.{ }^{3} \mathrm{H}\right]$ Para-aminohippuric acid $(4.3 \mathrm{Ci} / \mathrm{mmol})$ and $\left[{ }^{3} \mathrm{H}\right]$ estrone-3-sulfate $(60.6 \mathrm{Ci} / \mathrm{mmol})$ were purchased from PerkinElmer (Waltham, MA). $\left[{ }^{3} \mathrm{H}\right]$ Penciclovir $(1.1 \mathrm{Ci} / \mathrm{mmol})$ and $\left[{ }^{14} \mathrm{C}\right] \mathrm{metformin}(89.1 \mathrm{mCi} / \mathrm{mmol})$ were purchased from Moravek Biochemicals (Brea, CA). All other chemicals were of the highest purity available and purchased from Millipore Sigma (St. Louis, MO). Common human plasma and the plasma stripped three or four times with charcoal were purchased from Bioreclamation IVT (Westbury, NY). All cellculture media and reagents were obtained from Corning (Manassas, VA) or Thermo Fisher Scientific/Life Technologies (Carlsbad, CA). Sources of other materials are stated in their respective sections.

Clinical Samples from Drug Interaction Study between RIF and RSV in Healthy Subjects. Plasma samples were collected from a study of pharmacokinetic interaction between RIF and RSV that was reported previously (Lai et al., 2016). Briefly, twelve males, 18-45 years and with body mass index of 18.5$25.0 \mathrm{~kg} / \mathrm{m}^{2}$, participated in this open-labeled, three-period study. Each subject had a normal medical history, physical examination, and standard laboratory tests. Each subject received $600 \mathrm{mg}$ of RIF alone (period 1), $5 \mathrm{mg}$ of RSV alone (period 2), and $5 \mathrm{mg}$ of RSV after pretreatment with $600 \mathrm{mg}$ RIF (period 3). An interval of 1 week elapsed between studies. Subjects fasted the night before and until at least 4 hours after administration of the drugs. RIF and RSV were administered orally with $240 \mathrm{ml}$ of water. Blood samples $(3 \mathrm{ml})$ to determine the drug concentration were obtained at predose and at $0.5,1,1.5,2,2.5,3,4,6,8,12$, and 24 hours postdose. The plasma samples were prepared from the blood samples and were separated into two aliquots (Lai et al., 2016). The plasma samples from 8 out of 12 subjects, who gave informed consent and voluntarily agreed to additional assessments, were used to measure levels of BAs, DHEAS, HDA, and TDA.

Quantification of BAs in Plasma by LC-MS/MS. The bioanalytical analysis of BAs was performed as described previously (Zheng et al., 2016). Seven deuterium-labeled BAs, including cholic acid- $\mathrm{d}_{4}\left(\mathrm{CA}-\mathrm{d}_{4}\right)$, deoxycholic acid- $\mathrm{d}_{4}$ $\left(\right.$ DCA- $\left.\mathrm{d}_{4}\right)$, lithocholic acid- $\mathrm{d}_{4}\left(\right.$ LCA-d $\left.\mathrm{d}_{4}\right)$, glycoursodeoxycholic acid- $\mathrm{d}_{4}$ (GUDCA- $\left.\mathrm{d}_{4}\right)$, glycocholic acid- $\mathrm{d}_{4}\left(\right.$ GCA- $\left.\mathrm{d}_{4}\right)$, taurochenodeoxycholic acid- $\mathrm{d}_{5}$ $\left(\right.$ TCDCA- $\left.\mathrm{d}_{5}\right)$, and taurocholic acid- $\mathrm{d}_{5}\left(\mathrm{TCA}-\mathrm{d}_{5}\right)$, were used to generate calibration curve standards (Supplemental Table 1). Individual $1.0 \mathrm{mM}$ stocks were prepared by dissolving the appropriate amount of each standard in dimethyl sulfoxide. A $20,000 \mathrm{nM}$ plasma stock was made by pipetting $20 \mu \mathrm{l}$ of each of these seven $1 \mathrm{mM}$ standards into a 2.0-ml microcentrifuge tube (Eppendorf AG, Hamburg, Germany) containing $860 \mu \mathrm{l}$ of blank human plasma and vortexed. This pooled deuteriumlabeled BA sample was serial, diluted 1:1 with blank plasma to create 12 calibration standards covering a range from 4.9 to $20,000 \mathrm{nM}$. The internal standard (IS) solution was $2000 \mathrm{nM}$ dehydrocholic acid in acetonitrile.

Frozen samples were thawed at room temperature for 1 hour. A 96-well Strata Impact Protein Precipitation Plate (Phenomenex, Torrance, CA) was placed atop a 96-well collection plate (Eppendorf AG, Hamburg, Germany). A volume of $150 \mu \mathrm{l}$ IS solution and $40 \mu \mathrm{l}$ water was added to each well. Next, $100 \mu \mathrm{l}$ of plasma samples, calibration curve standards, quality control standards, or blanks were added to the corresponding wells. The plate combination was vortexed for 2 minutes at a speed of 1000 rpm using a DVX-2500 MultiTube Vortexer (VWR, 

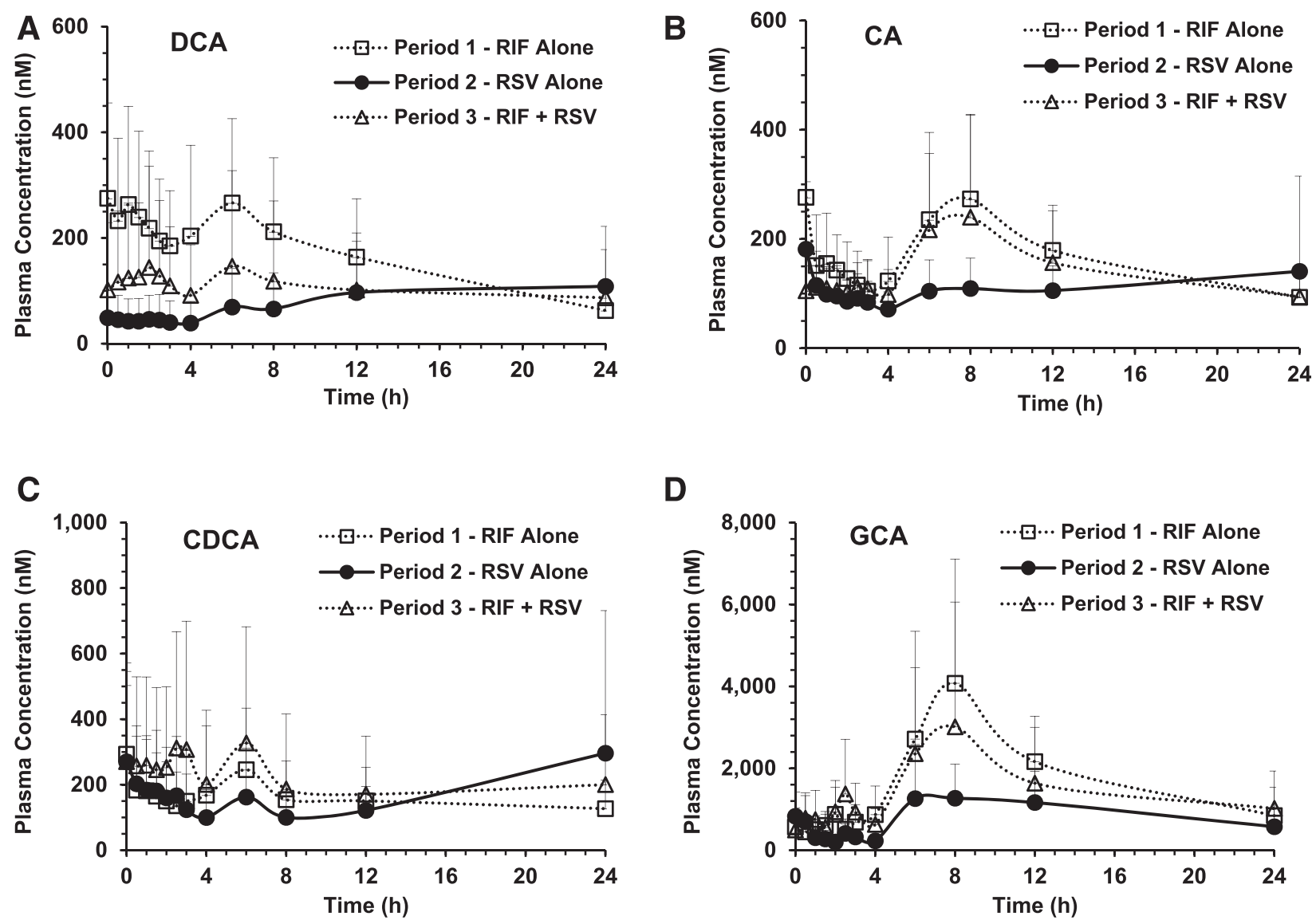

D
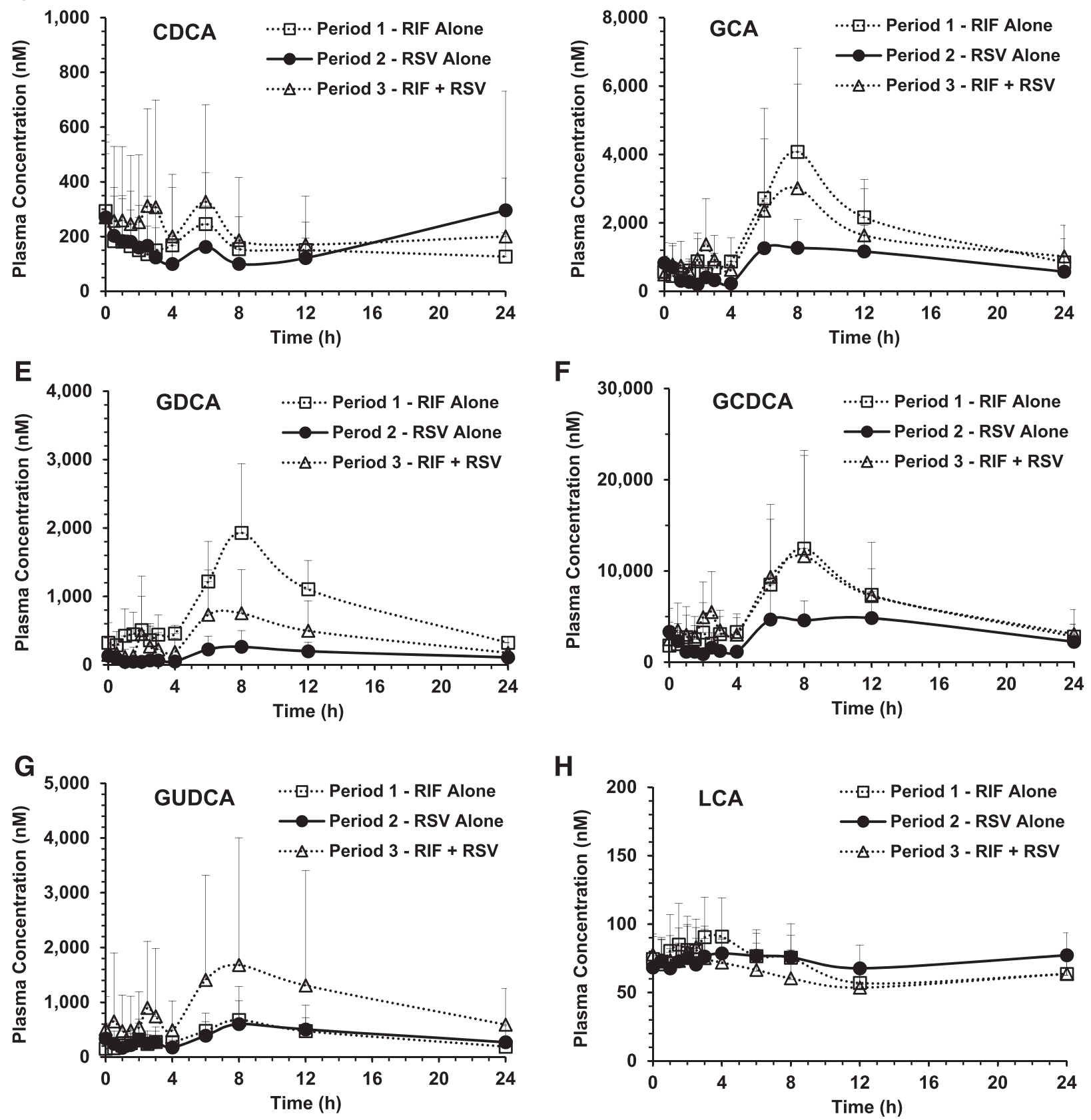

Fig. 1. Bile acid plasma concentrations (mean \pm S.D.; $n=7-8$ ) during RIF (open squares), RSV (solid circles), and coadministration of RIF and RSV (open triangles) periods. 

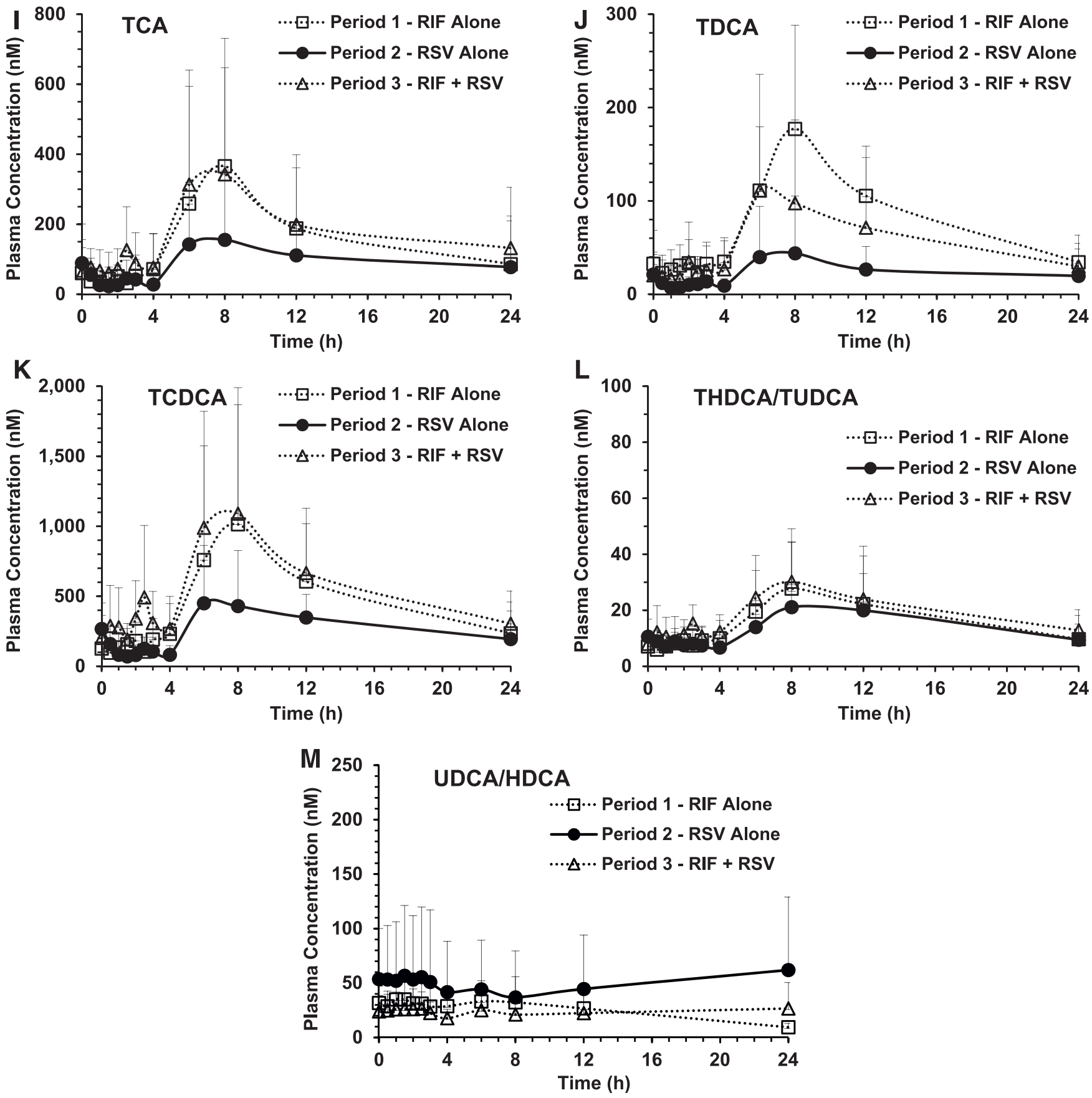

Fig. 1. Continued

Radnor, PA) to mix the samples and to initiate protein precipitation. The plate combination was centrifuged at a speed of $900 \mathrm{~g}$ for 5 minutes at $4^{\circ} \mathrm{C}$ (Eppendorf 5810R refrigerated centrifuge) to pull the supernatant through the filter into the collection plate. The filter plate was discarded, and $200 \mu \mathrm{l}$ of water was added to each well, which improved the chromatographic separation. The collection plate was capped, vortexed for 2 minutes at a speed of 1000 rpm using the DVX-2500 MultiTube Vortexer, and submitted to LC-MS/MS analysis.

The LC-MS/MS system consisted of an Acquity I-Class UPLC (Waters, Milford, MA) interfaced with a API6500 triple quadrupole mass spectrometer (AB Sciex, Toronto, ON, Canada). The LC and MS conditions were optimized using commercially available standards of common and deuterium-labeled BAs, for the simultaneous and unambiguous but also timely analysis of 24 analytes with two occasions where two analytes coelute (i.e., UDCA/HDCA and THDCA/ TUDCA). The samples were kept at $10^{\circ} \mathrm{C}$ in the autosampler and a $10-\mu 1$ volume was injected onto an Acquity BEH C18 column $(2.1 \times 50 \mathrm{~mm}, 1.7 \mu \mathrm{m}$; Waters $)$ at a temperature of $60^{\circ} \mathrm{C}$ with a flow rate of $0.7 \mathrm{ml} / \mathrm{min}$. The aqueous mobile phase $\mathrm{A}$ consisted of water with $0.1 \%$ formic acid and the organic mobile phase $\mathrm{B}$ consisted of acetonitrile with $0.1 \%$ formic acid. The mobile phase was held at $10 \%$ $\mathrm{B}$ for 0.3 minutes, a linear gradient was executed to $50 \% \mathrm{~B}$ at 1.9 minutes, then $50 \% \mathrm{~B}$ was held to 2.1 minutes, followed by another linear gradient to $90 \% \mathrm{~B}$ at 3.2 minutes, then $90 \% \mathrm{~B}$ was held to 3.5 minutes followed by a rapid return to $10 \% \mathrm{~B}$ at 3.6 minutes, which was followed by 0.4 minutes of re-equilibration at initial conditions for a total run time of 4 minutes.

The following source conditions were used for the mass spectrometer operation in electrospray negative ion mode: quadrupoles Q1 and Q3 at unit resolution, entrance potential $-5 \mathrm{~V}$, collision cell exit potential $-10 \mathrm{~V}$, curtain gas 35 units, collision gas nine units, ion spray voltage $-4500 \mathrm{~V}$, temperature $600^{\circ} \mathrm{C}$, ion source gas 1 of 50 units, and ion source gas 2 of 60 units. As shown in Supplemental 
Table 1, 24 precursor-to-product ion selected-reaction-monitoring (SRM) transitions were monitored using MS/MS detection with a dwell time of 10 milliseconds. The grouping of structurally similar BAs is depicted in Supplemental Table 1, where they are assessed against the same suitable deuterium-labeled BA. The data were acquired and analyzed using Analyst Instrument Control and Data Processing Software v. 1.6.2 (AB Sciex).

Quantification of DHEAS in Plasma by LC-MS/MS. Human plasma stripped three times with charcoal was used to create eight calibration standards covering a range from 68 to 50,000 nM. Plasma samples were extracted using organic protein precipitation. Specifically, $75 \mu \mathrm{l}$ of acetonitrile containing $2000 \mathrm{nM}$ DHEAS- $\mathrm{d}_{5}$ was added to $25 \mu$ plasma samples or calibration standards, followed by rigorous mixing on a mixer for 5 minutes at room temperature. The mixture was centrifuged at $4{ }^{\circ} \mathrm{C}$ for 10 minutes into a clean 96 -well receiving plate containing $50 \mu 1$ of water.

The LC-MS/MS analysis was performed on a Triple Quad 6500 (AB Sciex) coupled with a Shimadzu SCL 30AD Nexera liquid chromatography system, comprising two LC-30AD pumps and a Shimadzu SIL-30AC autosampler (Shimadzu, Kyoto, Japan). The chromatographic separation was performed on an Atlantis dC18 column $(2.1 \times 50 \mathrm{~mm}, 3 \mu \mathrm{m})$ from Waters. Mobile phase A was $10 \mathrm{mM}$ ammonium formate with $2 \%$ acetonitrile and mobile phase B was acetonitrile. A linear gradient was used to achieve chromatographic separation with the following linear gradients: 0.01 minutes, $10 \% \mathrm{~B} ; 2.5$ minutes, $75 \% \mathrm{~B}$; 2.6 minutes, $90 \% \mathrm{~B} ; 3.0$ minutes, $90 \% \mathrm{~B} ; 4.5$ minutes, $10 \% \mathrm{~B}$. A sample volume of $5 \mu \mathrm{l}$ was injected onto the $\mathrm{LC}$ column at a flow rate of $0.4 \mathrm{ml} / \mathrm{min}$. The analyte was monitored using multiple reaction monitoring (MRM) in electrospray negative ion mode. The turbospray voltage was set at $4500 \mathrm{~V}$ and turbo probe temperature was set at $450^{\circ} \mathrm{C}$; and declustering potential and collision energy were optimized to be $140 \mathrm{eV}$ and $36 \mathrm{eV}$. Quantitation was performed using the transitions of $\mathrm{m} / \mathrm{z} 367.1 \rightarrow 97.0$ and $72.1 \rightarrow 98.0$ for DHEAS and DHEAS- $\mathrm{d}_{5}$, respectively. The system control and data processing were performed on the Analyst v1.5.1 software.

Quantification of HDA and TDA in Plasma by LC-MS/MS. Human plasma stripped four times with charcoal was used to create seven reference standards covering a range from 4.1 to $1000 \mathrm{nM}$. Plasma samples were extracted using protein precipitation. Specifically, $75 \mu \mathrm{l}$ of methanol containing $200 \mathrm{nM}$ HDA- $\mathrm{d}_{28}$ and TDA- $\mathrm{d}_{24}$ was added to $25-\mu$ l plasma samples or calibration standards, followed by rigorous mixing on a mixer for 5 minutes at room temperature. The mixture was centrifuged at $4^{\circ} \mathrm{C}$ for 10 minutes into a clean 96-well receiving plate containing $50 \mu \mathrm{l}$ of water and subjected to LC-MS/MS analysis in a QTRAP 6500 mass spectrometer as described above. Chromatography was carried out with a Waters Atlantis dC18 column $(2.1 \times 50 \mathrm{~mm}$, $3 \mu \mathrm{m})$ employing mobile phase A (10 mM ammonium formate with $2 \%$ acetonitrile) and mobile phase $B$ (acetonitrile) at a flow rate of $0.6 \mathrm{ml} / \mathrm{min}$. The gradient program was set as follows: mobile phase B set at $10 \%(0$ 0.02 minutes), increase mobile phase B linearly from $10 \%$ to $60 \%(0.02-$ 3 minutes), increase mobile phase B linearly from $60 \%$ to $90 \%$ (3-3.2 minutes), maintain $90 \%$ mobile phase B (3.2-3.8 minutes), then decrease mobile phase B from $90 \%$ to $10 \%$. The mass spectrometer was operated with negative electrospray ionization and multiple reaction monitoring using the transitions of the protonated forms of HDA $(\mathrm{m} / z 285.1 \rightarrow 223.1)$, HDA- $\mathrm{d}_{28}(\mathrm{~m} / \mathrm{z} 313.2 \rightarrow$ 250.2), TDA $(\mathrm{m} / \mathrm{z} 257.2 \rightarrow 239.0)$, and TDA-d $\mathrm{d}_{24}(\mathrm{~m} / \mathrm{z} 281.2 \rightarrow 262.1)$. Optimized parameters were as follows: source temperature $500^{\circ} \mathrm{C}$ and ion spray voltage $4500 \mathrm{~V}$. Declustering potentials were $95,85,60$, and $80 \mathrm{~V}$ for HDA, HDA- $\mathrm{d}_{28}$, TDA, and TDA- $\mathrm{d}_{24}$, respectively. Collision energies were 36 , 34, 24 and $28 \mathrm{eV}$ for HDA, HDA-d28, TDA, and TDA-d24, respectively, and collision cell exit potential $9,15,13$, and $15 \mathrm{~V}$ for HDA, HDA- $\mathrm{d}_{28}$, TDA, and TDA- $\mathrm{d}_{24}$, respectively.

Uptake Studies in Transporter-Expressing Human Embryonic Kidney 293 Cells. Uptake of CPs and radiolabeled control substrates was measured using a protocol described previously (Shen et al., 2015; Shen et al., 2016). Briefly, cells were seeded in poly-D-lysine-coated 24 -well plates at a density of 500,000 cells per well and grown for 2-3 days in Dulbecco's modified Eagle's medium supplemented with $10 \%$ fetal bovine serum, $0.1 \mathrm{mM}$ nonessential amino acids, $2 \mathrm{mM}$ L-glutamate, and $100 \mu \mathrm{g} / \mathrm{ml}$ hygromycin. Medium was aspirated and cells were rinsed twice with prewarmed Hanks' balanced salt solution (HBSS). Uptake was initiated by the addition of prewarmed transport buffer (HBSS with $10 \mathrm{mM}$ HEPES, pH 7.4, for stably transfected OAT1-, OAT2-, OAT3-, OAT4-, and OCT2-HEK cells, and $\mathrm{pH} 8.4$ for MATE1- and MATE2K-HEK cells) containing CPs or radiolabeled probe substrate $(1$ or $2 \mu \mathrm{M})$. After a 5 -minute incubation, uptake was stopped by quick aspiration of the transport buffer followed by extensive washing with ice-cold HBSS. After drying the plate, cells were lysed with $0.1 \%$ Triton-X 100 or acetonitrile. Aliquots of cell lysate were used for radioactivity counting or LC-MS/MS measurement in addition to protein concentration analysis as described previously (Lai et al., 2016). Radioactivity was determined using a Tri-Carb 3100TR liquid scintillation counter (PerkinElmer).

TABLE 1

Summary statistics for $\mathrm{AUC}_{(0-24 \mathrm{~h})}$ of endogenous biomarkers in periods of RIF and RSV administration and coadministration of RIF and RSV

Values for $\mathrm{AUC}_{(0-24 \mathrm{~h})}$, area under plasma concentration-time curve from time 0 to 24 hours, are expressed as arithmetic means $\pm \mathrm{S} . \mathrm{D}$. $(\mathrm{n}=7-8)$. $A U C_{(0-24 \mathrm{~h})}$ ratios relative to the RSV period are geometric means $(90 \% \mathrm{CI})$.

\begin{tabular}{|c|c|c|c|c|c|}
\hline \multirow[b]{2}{*}{ Analyte } & \multicolumn{2}{|r|}{ RIF Period } & \multirow{2}{*}{$\begin{array}{l}\text { RSV Period } \\
\text { AUC }_{(0-24 \mathrm{~h})}\end{array}$} & \multicolumn{2}{|c|}{ Coadministration of RIF and RSV Period } \\
\hline & $\mathrm{AUC}_{(0-24 \mathrm{~h})}$ & $\begin{array}{c}\text { AUC }_{(0-24 h)} \text { Ratio } \\
\text { (Versus RSV Period) }\end{array}$ & & $\mathrm{AUC}_{(0-24 \mathrm{~h})}$ & $\begin{array}{c}\text { AUC }_{(0-24 h)} \text { Ratio } \\
\text { (Versus RSV Period) }\end{array}$ \\
\hline & $\mu M * h$ & Geometric Mean (90\% CI) & $\mu M * h$ & $\mu M * h$ & Geometric Mean $(90 \% \mathrm{CI})$ \\
\hline DCA & $3.8 \pm 2.3$ & $3.2(0.94,11)$ & $2.0 \pm 1.9$ & $2.5 \pm 2.7$ & $0.95(0.57,1.6)$ \\
\hline $\mathrm{CA}$ & $3.9 \pm 1.8$ & $1.6(1.3,1.9)$ & $2.7 \pm 1.2$ & $3.4 \pm 1.8$ & $1.3(0.97,1.8)$ \\
\hline CDCA & $3.7 \pm 2.3$ & $1.1(0.61,2.1)$ & $4.1 \pm 3.6$ & $5.0 \pm 4.7$ & $1.0(0.58,1.8)$ \\
\hline GCA & $4.1 \pm 2.1$ & $2.3(1.8,2.8)$ & $2.0 \pm 1.1$ & $3.5 \pm 3.0$ & $1.7(1.3,2.1)$ \\
\hline GDCA & $19.7 \pm 5.2$ & $7.4(3.1,17.4)$ & $3.7 \pm 2.7$ & $9.4 \pm 8.1$ & $1.9(1.0,3.7)$ \\
\hline GCDCA & $136.7 \pm 61.5$ & $1.8(1.5,2.2)$ & $78.6 \pm 40.1$ & $142.7 \pm 114.7$ & $1.6(1.3,2.0)$ \\
\hline GUDCA & $8.8 \pm 3.8$ & $1.1(0.61,2.2)$ & $9.2 \pm 8.6$ & $23.7 \pm 31.3$ & $1.9(0.81,4.6)$ \\
\hline LCA & $1.6 \pm 0.36$ & $0.90(0.78,1.0)$ & $1.8 \pm 0.33$ & $1.5 \pm 0.35$ & $0.86(0.75,0.99)$ \\
\hline TCA & $3.7 \pm 4.0$ & $2.0(1.6,2.6)$ & $2.2 \pm 2.7$ & $4.3 \pm 4.2$ & $2.2(1.5,3.2)$ \\
\hline TDCA & $1.8 \pm 0.79$ & $4.8(2.4,9.6)$ & $0.58 \pm 0.69$ & $1.3 \pm 1.3$ & $2.1(1.3,3.4)$ \\
\hline TCDCA & $11.0 \pm 7.9$ & $1.8(1.6,2.1)$ & $6.4 \pm 4.3$ & $13.4 \pm 9.0$ & $2.0(1.5,2.7)$ \\
\hline THDCA/TUDCA & $0.38 \pm 0.19$ & $1.4(0.82,2.4)$ & $0.33 \pm 0.26$ & $0.45 \pm 0.26$ & $2.1(1.6,2.8)$ \\
\hline UDCA/HDCA & $0.56 \pm 0.30$ & $0.66(0.31,1.4)$ & $1.2 \pm 1.2$ & $0.56 \pm 0.34$ & $0.97(0.66,1.4)$ \\
\hline DHEAS & $92.3 \pm 48.2$ & $0.97(0.89,1.1)$ & $101.5 \pm 45.5$ & $97.1 \pm 44.4$ & $1.0(0.95,1.1)$ \\
\hline HDA & $1.9 \pm 0.82$ & $2.2(1.8,2.6)$ & $0.90 \pm 0.37$ & $2.2 \pm 0.53$ & $2.5(2.1,3.0)$ \\
\hline TDA & $1.3 \pm 0.51$ & $2.8(2.2,3.5)$ & $0.47 \pm 0.26$ & $1.5 \pm 0.49$ & $3.2(2.4,4.2)$ \\
\hline $\mathrm{CP} \mathrm{I}^{a}$ & $0.084 \pm 0.019$ & $4.0(3.7,4.5)$ & $0.021 \pm 0.005$ & $0.084 \pm 0.016$ & $4.0(3.7,4.3)$ \\
\hline CP III $^{a}$ & $0.013 \pm 0.004$ & $3.4(2.9,4.1)$ & $0.004 \pm 0.001$ & $0.013 \pm 0.003$ & $3.5(3.0,4.0)$ \\
\hline $\mathrm{RSV}^{a}$ & & & $0.08 \pm 0.03$ & $0.37 \pm 0.12$ & $4.8(4.0,5.7)$ \\
\hline
\end{tabular}

CI, confidence interval.

${ }^{a}$ Data on plasma CP I, CP III, and RSV concentrations in healthy subjects are from a previous publication (Lai et al., 2016). 


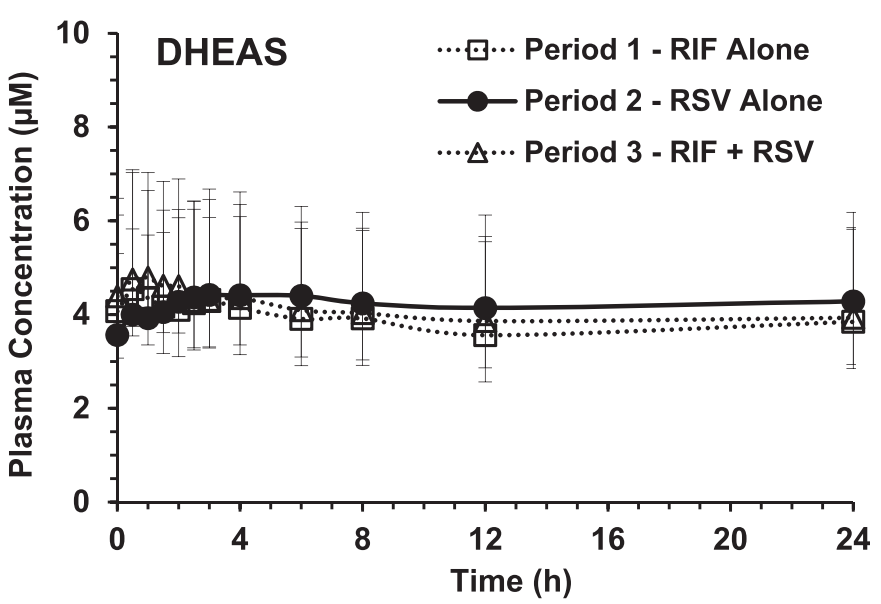

Fig. 2. DHEAS plasma concentrations (mean \pm S.D.; $n=7-8$ ) during RIF (open squares), RSV (solid circles), and coadministration of RIF and RSV (open triangles) periods.

Data Analysis for In Vivo Studies. Data are expressed in mean \pm S.D. Area under plasma concentration-time curve from time 0 to 24 hours [AUC $(0-24 \mathrm{~h})]$ for BAs, DHEAS, HDA, and TDA, following single oral administration of RIF and RSV with or without coadministration of RIF were analyzed with a mixed log-linear trapezoidal model using the Kinetica program (Thermo Electron, Philadelphia, PA). The $90 \%$ confidence intervals for the differences in $\mathrm{AUC}_{(0-24 \mathrm{~h})}$ between treatments on the natural $\log$ scale were exponentiated to obtain estimates for ratios of geometric means on the original scale.

To evaluate the effects of RIF treatment on plasma concentrations of BAs, DHEAS, HDA, TDA, and CPs I and III, a linear mixed-effects repeated measurements analysis of variance (ANOVA) model was applied on the $\log$ biomarker concentrations using SAS PROC MIXED procedure version 9.2 (SAS Institute, Cary, $\mathrm{NC}$ ). The model included treatment, time, and treatment-by-time (Treatment*Time) interaction terms as fixed effects, and subject as a random effect for describing the subject-to-subject variability in the data. Using the repeated measurements model, the changes in mean concentration profiles of the groups over the time are evaluated and compared by accounting for the betweensubject variance and within-subject variance and covariance over the time.

To compare and rank the sensitivities across different endogenous biomarkers, standardized effect size $(\theta)$ of RIF and RIF plus RSV treatment groups compared with RSV group on the $\mathrm{AUC}_{(0-24 \mathrm{~h})}$ was obtained as a measure of strength of the different biomarkers in terms of the relative magnitude of treatment effects from the repeated measurements model analysis. Specifically, the standardized effect size $\theta$ is computed as follows:

$$
\theta=\frac{\mu 1-\mu 2}{\sigma}
$$

where $\mu 1$ is the mean of RIF or RIF plus RSV treatment group, $\mu 2$ is the mean of RSV treatment, and $\sigma$ is a standard error of the mean difference.

Data Analysis for In Vitro Experiments. The in vitro uptake experiments were repeated in at least two independent experiments. Result shown are the mean \pm S.D. of triplicates run in one experiment. The calculated amounts of compound taken up by HEK cells during 5-minute incubation in the absence and presence of transporter inhibitor were graphed with mean data. Two-sided unpaired Student's $t$ test was performed to assess whether the uptake by transporter-expressing cells is significantly different from that by mock cells, and a $p$-value of less than 0.05 was considered statistically significant (GraphPad Software; San Diego, CA).

\section{Results}

\section{Effects of RIF Administration on Plasma BA Levels}

Figure 1 shows for the whole study period the arithmetic means \pm S.D. of plasma concentrations of 13 bile acids that include deoxycholic acid (DCA), cholic acid (CA), chenodeoxycholic acid (CDCA), glycocholic acid (GCA), glycodeoxycholic acid (GDCA), glycochenodeoxycholic acid (GCDCA),
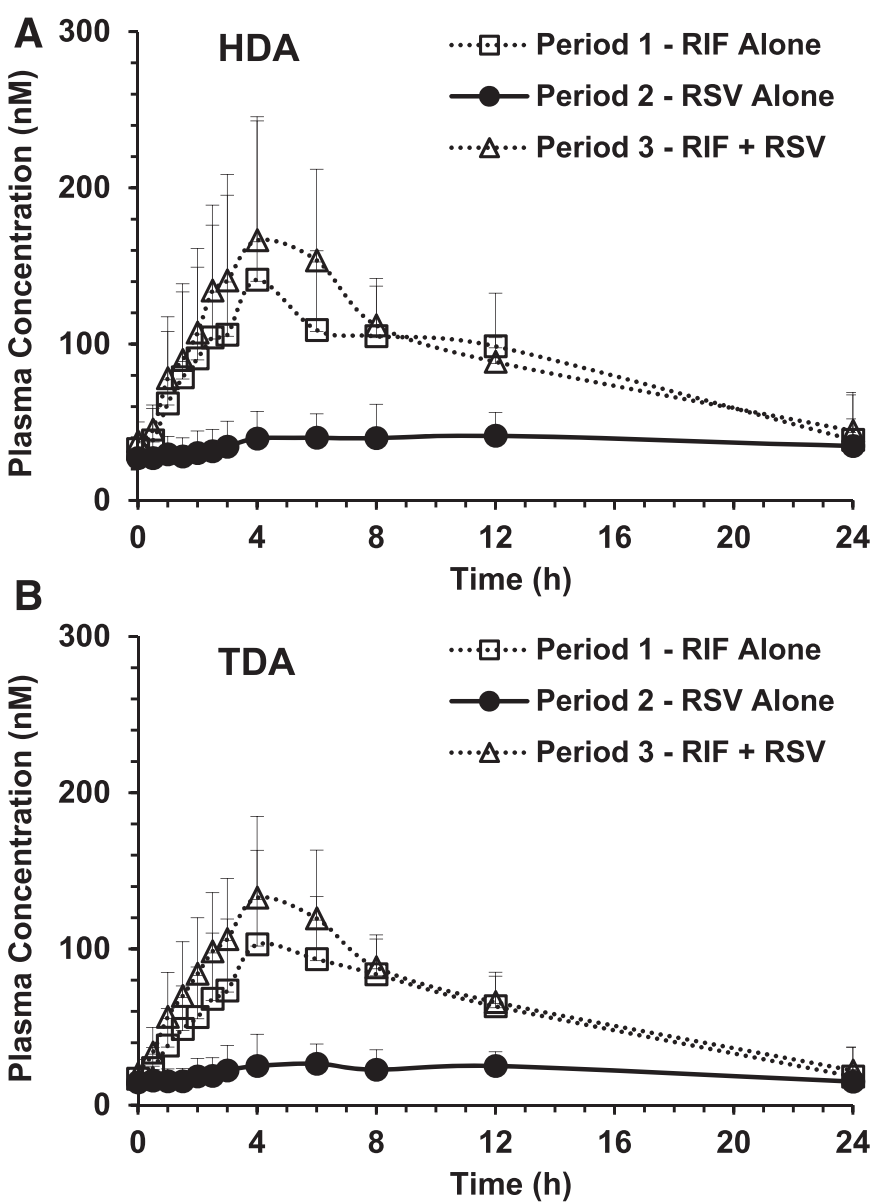

Fig. 3. HDA and TDA plasma concentrations (mean \pm S.D.; $n=7-8$ ) during RIF (open squares), RSV (solid circles), and coadministration of RIF and RSV (open triangles) periods.

glycoursodeoxycholic acid (GUDCA), lithocholic acid (LCA), taurocholic acid (TCA), taurodeoxycholic acid (TDCA), taurochenodeoxycholic acid (TCDCA), taurohyodeoxycholic acid/tauroursodeoxycholic acid (THDCA/ TUDCA), and ursodeoxycholic acid hyodeoxycholic acid (UDCA/HDCA). Supplemental Table 2 shows the arithmetic means \pm S.D. of plasma bile acid concentrations during the three periods. Administration of a single dose of $5 \mathrm{mg}$ RSV did not significantly alter plasma DCA, CA, CDCA, LCA, and UDCA/HDCA concentrations compared with predose baseline (Supplemental Table 2). In contrast, the levels of four glycine-conjugated bile acids (GCA, GDCA, GCDCA, and GUDCA) and four taurine-conjugated bile acids (TCA, TDCA, TCDCA, and THDCA/TUDCA) were increased from 4 to 12 hours after administration of RSV alone compared with the predose levels. Remarkable increases in the plasma levels of the eight BAs were detected at a time approximating the time of the first postdose meal (Fig. 1, D-G and I-L; Supplemental Table 2).

Plasma bile acid concentrations did not show statistically significant increase in RIF or RIF plus RSV group compared with RSV group when analyzed using the repeated measurement model ( $p$ value $>0.05$ ) even through GDCA and TDCA $\mathrm{AUC}_{(0-24 \mathrm{~h})}$ geometric mean ratio increased 1.9- to 7.4-fold (Fig. 1, F and J; Table 1). There were modest changes in DCA, CA, GCA, GUDCA, GCDCA, TCA, and TCDCA concentrations after administration of RIF or RIF plus RSV (Fig. 1, A, B, D, F, I, and K). There were no changes in CDCA, LCA, THDCA/TUDCA, and UDCA/ HDCA concentrations after administration of RIF or RIF plus RSV (Fig. 1, $\mathrm{C}, \mathrm{H}, \mathrm{L}$, and $\mathrm{M}$ ) (Table 1). 


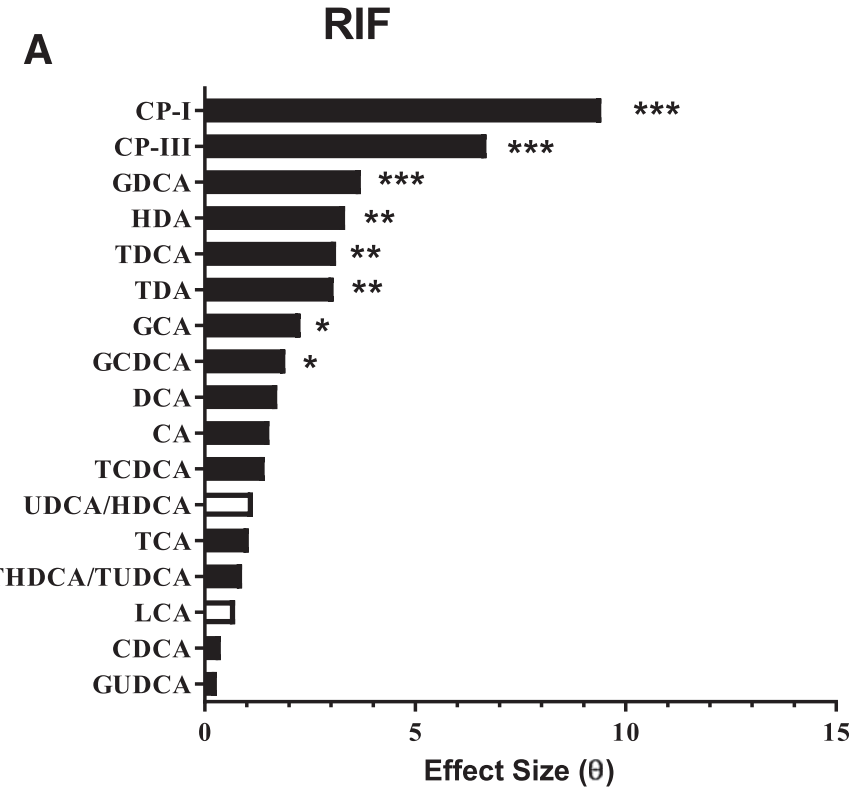

B

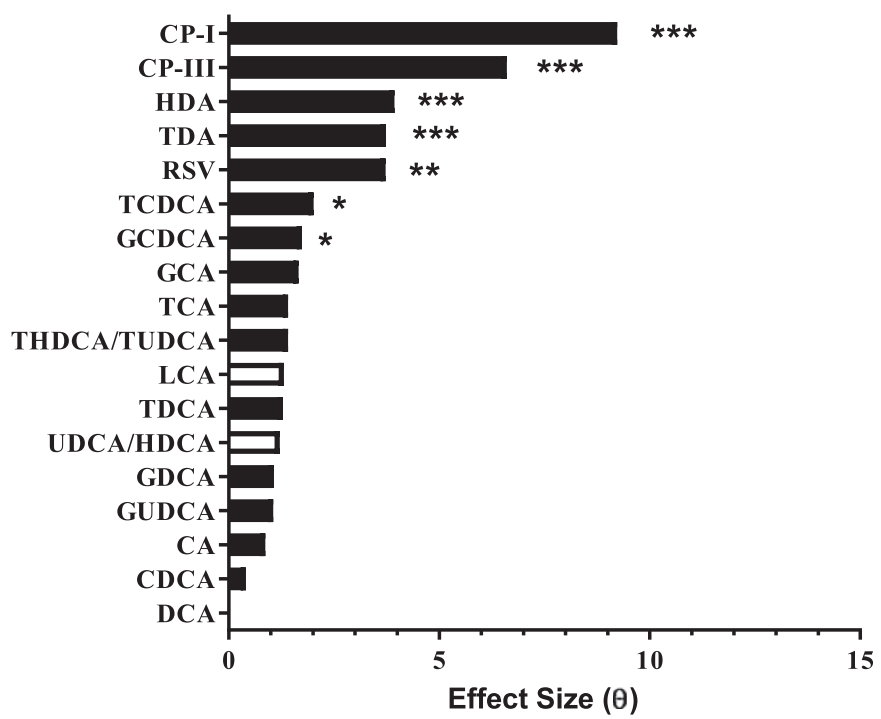

Fig. 4. Comparison of sensitivities of various endogenous biomarkers of OATP activity in the treatments with RIF (A) or RIF plus RSV (B) in eight human subjects. The biomarkers were ranked by comparing $\mathrm{AUC}_{(0-24 \mathrm{~h})}$ using the effect-size approach (see Materials and Methods for the details) $(* * * p<0.0001, * * p<0.01$, and $* p<0.05)$. The black bars represent positive values and white bars represent negative.

\section{Effects of RIF Administration on Plasma DHEAS Levels}

Figure 2 displays the arithmetic means \pm S.D. of plasma concentrations of DHEAS for the entire study period. DHEAS values were comparable and not statistically different among RIF, RSV, and RIF plus RSV periods, indicating no major effect of RIF on DHEAS (Fig. 2) (Table 1 and Supplemental Table 3).

\section{Effects of RIF Administration on Plasma HDA and TDA Levels}

Results comparing systemic exposures of HDA and TDA are shown in Fig. 3, Table 1, and Supplemental Table 4. After administration of RIF alone or RIF plus RSV, plasma HDA and TDA increased approximately 2.2- to 3.2-fold higher than RSV period $\left[\mathrm{AUC}_{(0-24 \mathrm{~h})}\right.$ ratios of $2.2(1.8$,
2.6) and $2.5(2.1,3.0)$, and $2.8(2.2,3.5)$ and $3.2(2.4,4.2)$ for HDA and TDA, respectively] (Table 1). The increases were statistically significant $(p<0.05)$ (Fig. 3). The magnitude of changes in HDA and TDA AUC $(0-24 \mathrm{~h})$ was slightly less than that of RSV (2.2- to 3.2-fold versus 4.8-fold).

To compare the effects of RIF on various biomarkers with respect to change in $\mathrm{AUC}_{(0-24 \mathrm{~h})}$, the relative effect size values to $\operatorname{RSV}(\theta)$ were computed. As shown in Fig. 4, the overall ranking of sensitivity of endogenous biomarkers following RIF treatment are $\mathrm{CP}$ I $>\mathrm{CP}$ III $>$ $\mathrm{HDA} \approx \mathrm{TDA} \approx \mathrm{RSV}>>$ BAs.

Involvement of Renal Transporters in CPs I and III Transport. Previously we demonstrated that CPs I and III are substrates of OATP1B1 and OATP1B3, respectively (Shen et al., 2016). To further assess substrate specificity of CPs I and III, we performed in vitro substrate profiling of CPs I and III for renal transporters, including OAT1, OAT2, OAT3, OAT4, OCT2, MATE1, and MATE2K, using transporter-overexpressing cells. Uptake of $1 \mu \mathrm{M}$ CPs I and III by OAT1-, OAT2-, OAT3-, or OAT4-HEK cells was not significantly statistically different from that by mock-HEK cells (Supplemental Fig. 1). Similarly, the uptake of CPs I and III by the cells stably expressing OCT2, MATE1 and MATE2K was not significantly different from mock-HEK cells (Supplemental Fig. 2). In contrast, cells expressing individual transporter consistently demonstrated significantly higher uptake of radiolabeled probe substrate compared with mock-HEK cells. In addition, complete inhibition of the uptake of probe substrates by typical transporter inhibitors was observed (Probenecid for OAT, and pyrimethamine and quinidine for OCT2 and MATEs, respectively). These results clearly suggest that CPs I and III are not substrates of renal transporters OAT1, OAT2, OAT3, OAT4, OCT2, MATE1, and MATE2K.

Inhibitory Effects of RIF on Transport of CPs I and III by Hepatic OATP Transporters. To assess the potential effect of substrate difference on the inhibitory interaction of RIF with hepatic OATP transporters, a parallel set of $\mathrm{IC}_{50}$ values was generated against transport of CPs I and III in comparison with those against RSV reported previously by us and others. CPs I and III concentrations $(0.2 \mu \mathrm{M})$ were selected on the basis of a balance of low Michaelis constant values and sensitivity limits of the analytical assays. Figure 5 shows that increasing concentrations of RIF-inhibited OATP1B1-mediated transport of both CPs I and III and the $\mathrm{IC}_{50}$ values did not differ significantly $(0.66 \pm 0.11$ and $0.79 \pm 0.16 \mu \mathrm{M}$, respectively). They were also not different from the $\mathrm{IC}_{50}$ for uptake of RSV (Table 2) (Shen et al., 2013; Prueksaritanont et al., 2014; Izumi et al., 2015). We further investigated the relationship between the $\mathrm{IC}_{50}$ values for the inhibition of OATP1B3- and OATP2B1mediated $\mathrm{CP}$ transport and the $\mathrm{IC}_{50}$ values for the inhibition of OATP1B3- and OATP2B1-mediated RSV transport reported in literature. Again, RIF showed comparable $\mathrm{IC}_{50}$ values between substrates for OATP1B1 and OATP2B1 inhibition, and less than 2-fold difference was generally observed (Figs. 6 and 7; Table 2). It is worth noting that CP I uptake by OATP2B1-HEK cells was not significantly different from that by mock-HEK cells, whereas there was significant uptake of CP III by OATP2B1-HEK cells compared with mock-HEK cells and coincubation with RIF diminished the uptake of CP III to the control level (Fig. 7). This indicates that CP III but not CP I is a substrate for OATP2B1.

\section{Discussion}

Prediction of OATP-mediated drug-drug interactions present an inherent challenge given the difficulty in quantitative in vitro-to-in vivo extrapolation primarily as a result of challenges in determining the effective concentration of an NCE available at the site of OATP inhibition. Thus, endogenous substrates are attractive indicators reflecting interactions of an NCE with OATP in vivo. Importantly, the selection 

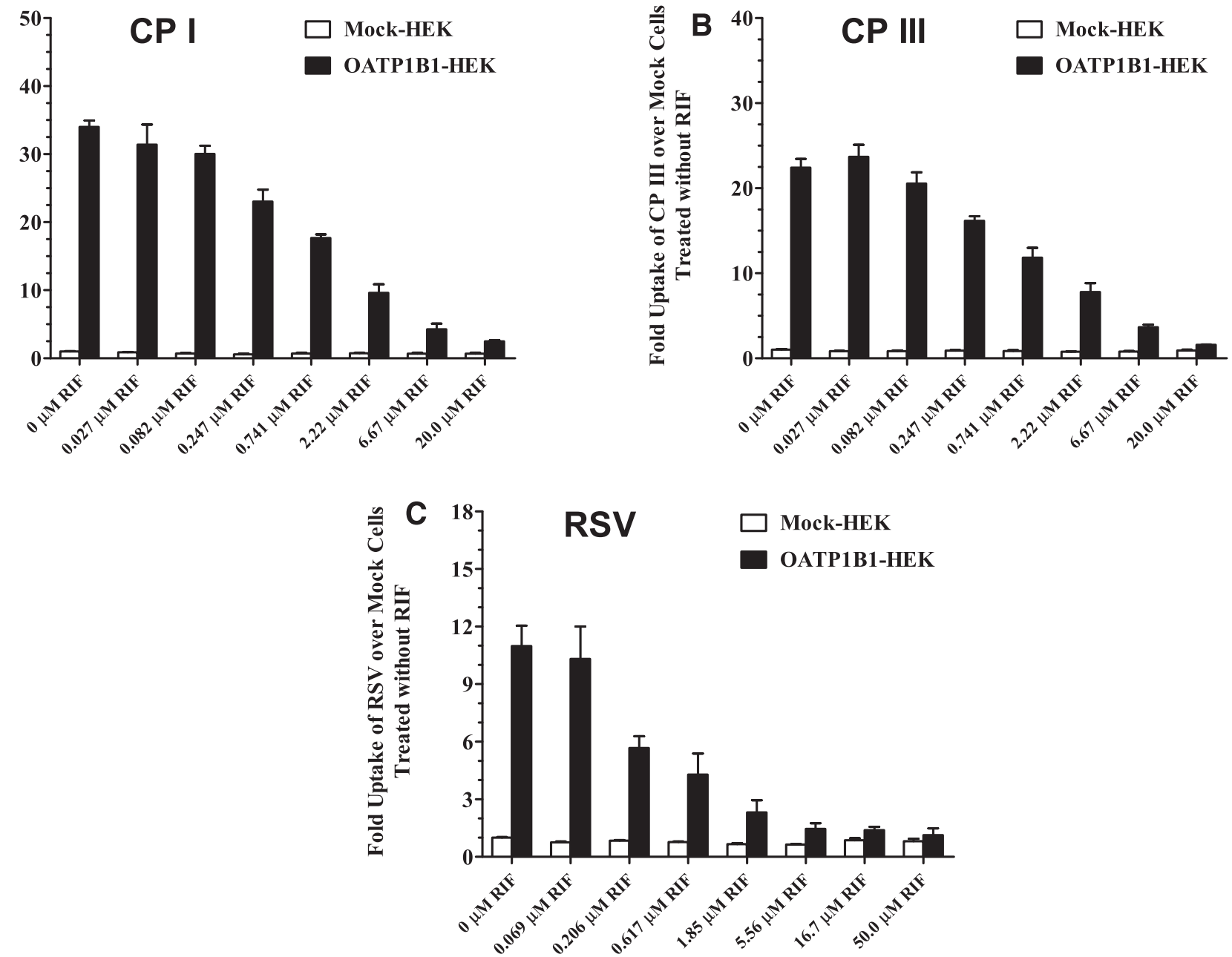

Fig. 5. Inhibitory effect of RIF on OATP1B1-mediated transport of CP I (A), CP III (B), and RSV (C). Uptake of $0.2 \mu \mathrm{M}$ CP I, $0.2 \mu \mathrm{M}$ CP III, and $1 \mu \mathrm{M}$ RSV was assessed in stably transfected OATP1B1-HEK cells over 2 minutes in the absence (control) or presence of RIF at the indicated concentrations. Fold changes are normalized to mockHEK cell uptake in the absence of RIF. Results are the mean \pm S.D. of three separate determinations.

of an endogenous substrate as a transporter biomarker requires that the marker is not only sensitive to the drug interaction but also specific to the transporter of interest in order to exclude the potential for additional interactions with other transporters. In addition, the association of markers with circadian rhythm, diet and disease, or organ injury should be carefully considered. OATPs (1B1 and 1B3) are involved in the transport of many endogenous compounds such as bile acids, hormone metabolites, bilirubin, and fatty acids. To date, of the potentially useful
OATP biomarkers identified in vitro, only CPs I and III have been shown to be useful for an early assessment of in vivo inhibition properties of a drug candidate (Lai et al., 2016). In addition to the CPs, several other endogenous substrates of OATP have been proposed as potential markers reflecting OATP function on the basis of the in vivo preclinical results or human pharmacogenetics analysis (Chu et al., 2015; Shimada et al., 2015a, b; Takahashi et al., 2015; Yee et al., 2016). To assess the performance of these endogenous substrates as biomarkers for OATP

TABLE 2

Comparison of $\mathrm{RIF} \mathrm{IC}_{50}$ values for inhibition of CP I, CP III, and RSV transport by hepatic OATP transporters

Data are expressed as means \pm S.D. $(n=3)$.

\begin{tabular}{|c|c|c|c|}
\hline \multirow{2}{*}{ Transporter } & \multicolumn{3}{|c|}{$\operatorname{RIF} \mathrm{IC}_{50}(\mu \mathrm{M})$} \\
\hline & CP I $(0.2 \mu \mathrm{M})$ & CP III $(0.2 \mu \mathrm{M})$ & RSV (Reference) \\
\hline OATP1B1 & $0.66 \pm 0.11$ & $0.79 \pm 0.16$ & $\begin{array}{c}1.10 \pm 0.28 \text { (Shen et al., 2013); } \\
1.1 \pm 0.2 \text { (Prueksaritanont et al., 2014) } \\
0.95 \pm 0.10 \text { (Izumi et al., 2015) }\end{array}$ \\
\hline OATP1B3 & $0.25 \pm 0.03$ & $0.27 \pm 0.02$ & $\begin{array}{c}0.49 \pm 0.13 \text { (Shen et al., 2013) } \\
0.3 \pm 0.05 \text { (Prueksaritanont et al., 2014) } \\
0.2 \pm 0.1(\text { Chu et al., 2015) }\end{array}$ \\
\hline $\mathrm{OATP} 2 \mathrm{~B} 1^{a}$ & No uptake & $48.4 \pm 7.6$ & $\begin{array}{c}89.8 \pm 5.4 \text { (Shen et al., 2013) } \\
81 \pm 34 \text { (Prueksaritanont et al., 2014) }\end{array}$ \\
\hline
\end{tabular}

${ }^{a}$ Significant uptake of CP III but not CP I by OATP2B1-HEK cells versus mock-HEK cells. 

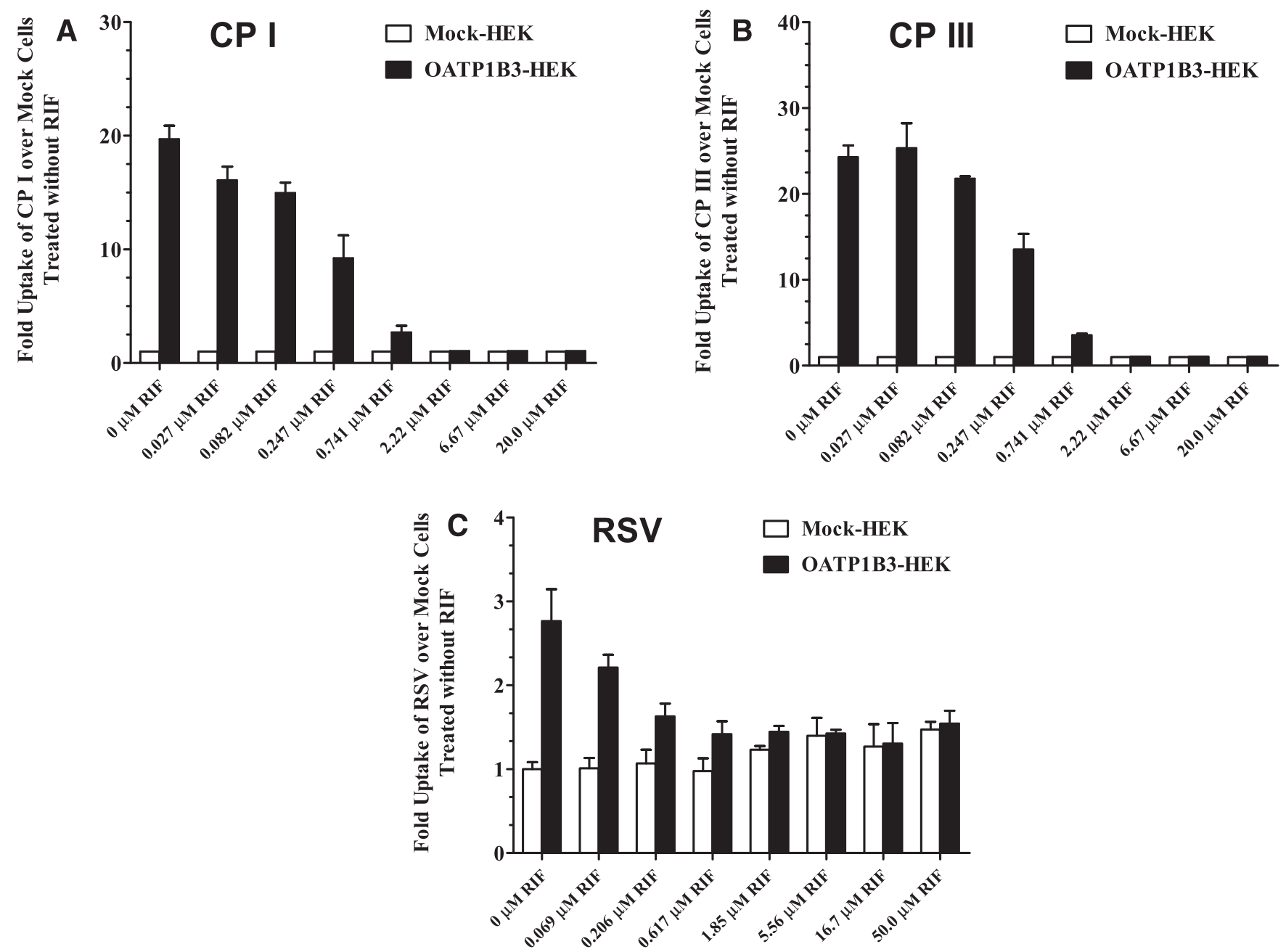

Fig. 6. Inhibitory effect of RIF on OATP1B3-mediated transport of CP I (A), CP III (B), and RSV (C). Uptake of $0.2 \mu \mathrm{M} \mathrm{CP} \mathrm{I,} 0.2 \mu \mathrm{M} \mathrm{CP} \mathrm{III,} \mathrm{and} 1 \mu \mathrm{M}$ RSV was assessed in stably transfected OATP1B1-HEK cells over 2 minutes in the absence (control) or presence of RIF at the indicated concentrations. Fold changes are normalized to mockHEK cell uptake in the absence of RIF. Results are the mean \pm S.D. of three separate determinations.

inhibition, we further measured the other less well characterized candidate markers, i.e., plasma BAs, DHEAS, HDA, and TDA in human samples and compared them with previously determined changes of CPs and RSV when RIF is coadministered (Lai et al., 2016).

When studying BAs as probes to reflect possible alterations in OATP activity at the therapeutic RIF dose of $600 \mathrm{mg}$, no statistically significant increases in plasma concentrations of BAs were observed even though some of them, such as GDCA, TDCA, DCA, GCA, and TCA, exhibited increased $\mathrm{AUC}_{(0-24 \mathrm{~h})}$ ratio by $\mathrm{RIF}$ (Fig. 1). This may be attributed to variation in plasma BA levels that are sensitive to the ingestion of meals (Ponz De Leon et al., 1978; Everson, 1987). In addition, the maximal postprandial increase of serum BA in patients with hepatic diseases has been found to be higher than healthy subjects, and the duration of serum BA elevation was significantly prolonged (Fausa, 1976; Engelking et al., 1980). Such large fluctuation in plasma/serum concentrations induced by diet and disease could certainly complicate the interpretation of results for BAs in a DDI study. Indeed, despite similar AUC changes between GDCA or TDCA and RSV (1.9- to 7.4-fold versus 4.8-fold) (Table 1), plasma GDCA and TDCA demonstrated a high degree of variation in the RSV period (control period) (Fig. 1). Furthermore, besides OATP1B, NTCP plays an important role in bile acid uptake from portal blood into the liver (Meier and Stieger, 2002). It has been reported that RIF is a more potent inhibitor of OATP1B1 and OATP1B3 than
NTCP ( IC $_{50}$ values of $0.2-1.1 \mu \mathrm{M}$ versus $277 \mu \mathrm{M}$ ) (Prueksaritanont et al., 2014; Chu et al., 2015). Although it was statin probes that were used in those OATP inhibition assessments and substrate-dependent OATP inhibition might have existed (Izumi et al., 2013), the results suggested that GDCA and TDCA are probably transported by OATP1B rather than NTCP. However, as these bile acids are "dual" substrates for both NTCP and OATP1B, it would be difficult to tease out the role of OATP1B from that of NTCP in BA uptake inhibition if an NCE is the dual inhibitor. In addition, other intestinal and hepatic transporters, including apical sodium-dependent bile acid transporter, organic solute transporters, ATP-dependent bile salt excretory pump (BSEP), and multidrug-resistant proteins play a critical role in maintaining enterohepatic circulation and bile acid homeostasis (Alrefai and Gill, 2007; Dawson et al., 2009). Inhibition of any of those transporters, such as BSEP, may also increase plasma BA concentrations. Taken together, our findings suggest that bile acids, including GCDA and TCDA, are probably not optimal indicators for OATP inhibition. However, although sulfated bile acids were only $1 \%$ of the plasma bile acid pool, there is a very recent report that a robust RIF dose-response, up to 50-fold increase in plasma AUC, was observed for the sulfates of five bile acids (GDCA-S, GCDCA-S, TCDCA-S, DCA-S, and TDCA-S) in cynomolgus monkeys (Thakare et al., 2017). It is envisioned that these probes will be increasingly studied as human OATP substrates and compared with other biomarkers. 

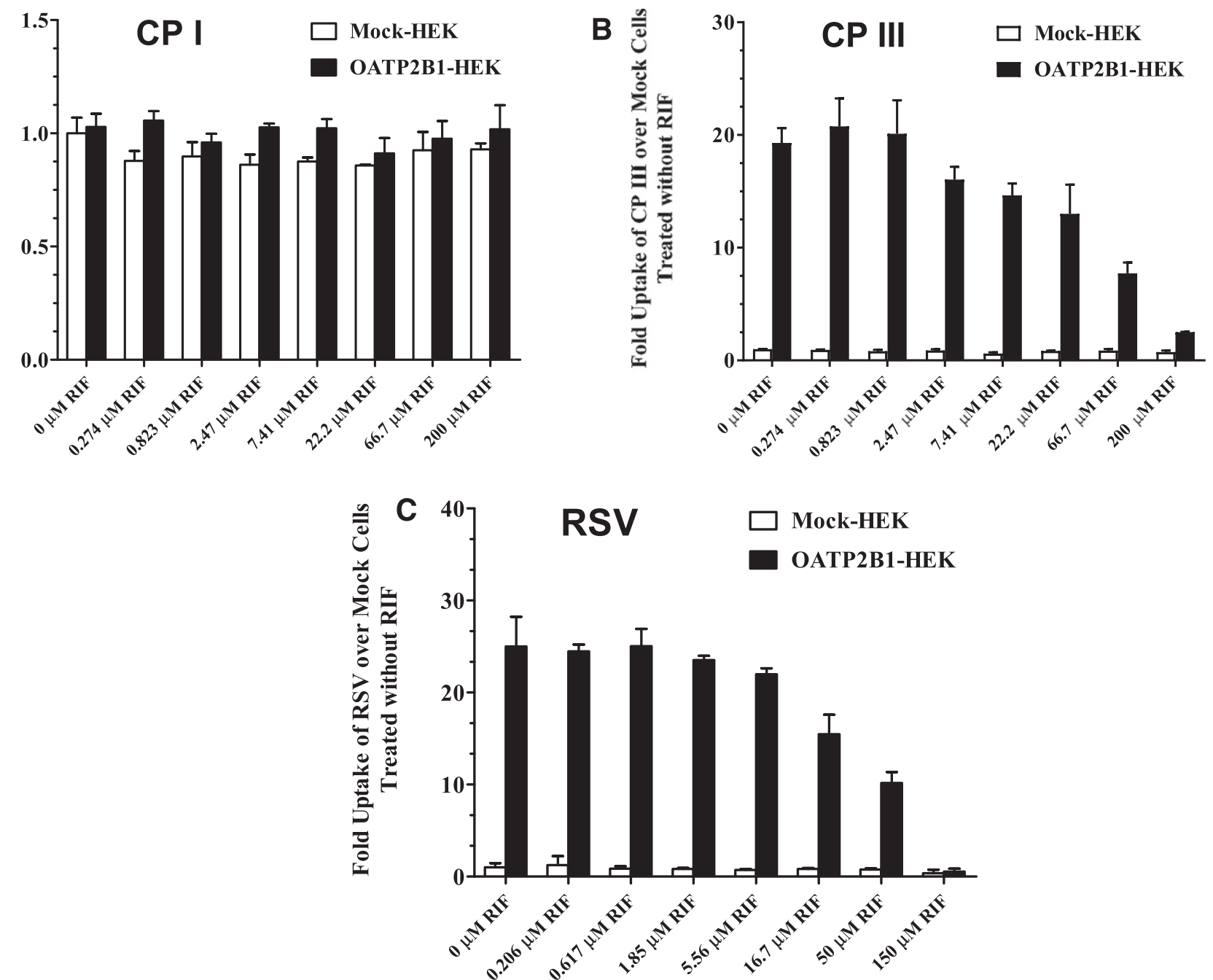

Fig. 7. Inhibitory effect of RIF on OATP2B1-mediated transport of CP I (A), CP III (B), and RSV (C). Uptake of $0.2 \mu \mathrm{M}$ CP I, $0.2 \mu \mathrm{M}$ CP III, and $1 \mu \mathrm{M}$ RSV was assessed in stably transfected OATP1B1-HEK cells over 2 minutes in the absence (control) or presence of RIF at the indicated concentrations. Fold changes are normalized to mockHEK cell uptake in the absence of RIF. Results are the mean \pm S.D. of three separate determinations.

Healthy subjects treated with $600 \mathrm{mg}$ RIF demonstrated no change in plasma DHEAS concentration compared with RSV treatment (Fig. 2 and Table 1). This is in contrast to earlier in vitro and in vivo findings in monkeys (Kullak-Ublick et al., 2001; Koenen et al., 2012; Watanabe et al., 2015). The time- and temperature-dependent uptake of DHEAS in human and monkey hepatocytes were observed. RIF significantly reduced the uptake, suggesting that OATP1B1 and OATP1B3 play an important role in DHEAS uptake (Watanabe et al., 2015). Plasma DHEAS level was significantly increased by 2 and $10 \mathrm{mg} / \mathrm{kg}$ RIF, although it was to a lower extent than the RSV increase in cynomolgus monkeys. Species differences in synthesis, disposition, and elimination of DHEAS may partly explain the discrepancy. For example, the synthesis and secretion rate of dehydroepiandrosterone and DHEAS from the zona reticularis in humans is higher than animal models used in the laboratory, including guinea pigs, mice, and rats (Labrie et al., 2005).

Another finding of the present study is the correlation of RIF-induced increases in plasma concentrations between HDA and TDA with RSV in healthy subjects $\left(\mathrm{AUC}_{(0-24 \mathrm{~h})}\right.$ ratios of 2.2- to 3.2-fold versus 4.8 -fold) (Fig. 3 and Table 1), suggesting that the endogenous HDA and TDA could be used as markers for OATP inhibition in clinical investigation. Our results confirm an earlier study reporting significantly higher correlations between HDA and TDA with OATP1B1 genetic variants in genome-wide association studies and significant difference in plasma HDA and TDA after coadministration of CsA versus pravastatin alone (Yee et al., 2016). However, although Yee and colleagues compared plasma concentrations at a single time point (30 minutes) after the drug was administered and found a marginal increase of both plasma HDA and TDA, the present analysis showed changes in plasma concentrations of HDA and TDA over the entire time course of RIF exposure (Fig. 3). Notably, plasma HDA and TDA concentrations remained relatively stable between baseline and RSV treatment, suggesting lack of apparent diet effect and circadian disruption in healthy subjects, which is similar to CPs I and III (Lai et al., 2016). The results suggested that HDA and TDA in plasma can probably be markers to reflect OATP inhibition and its associated DDI. However, a caution should also be raised, as HDA and TDA were found to be associated with drug-metabolizing enzyme CYP4A11 and renal transporters OAT1 and OAT3, reflecting complexity of HDA and TDA disposition and potential difficulty in using them as OATP-specific endogenous biomarkers, in particular when a perpetrator drug inhibits multiple transporters (Yee et al., 2016). It is also worth noting that, although the functions of HDA and TDA are not fully understood, the serum concentrations of HDA, TDA, and other dicarboxylic acids are increased in patients with diseases, such as Reye syndrome, caused by an insult to fatty acid 
metabolism and (or) stimulation of omega-oxidation (Tonsgard, 1986, 1988).

Further comparative analysis was conducted for the endogenous probes that show promise as biomarkers of OATP activity, using a standardized effect-size approach on the overall exposure $\mathrm{AUC}_{(0-24 \mathrm{~h})}$. The overall effect sizes were ranked as follows: $\mathrm{CPI}>\mathrm{CP}$ III $>\mathrm{HDA} \approx$ $\mathrm{TDA} \approx \mathrm{RSV}>>$ BAs (Fig. 4), reconfirming that CPs I and III are the most sensitive indicators among the endogenous compounds examined. Although fold changes in plasma $\mathrm{AUC}_{(0-24 \mathrm{~h})}$ of some bile acids, such as TDCA and GDCA, with RIF treatments were comparable to the changes in CPs, HDA, and TDA, the effect size of RIF on the bile acids was lower than on other markers owing to the high variations over time, suggesting bile acids are probably not as robust as the other endogenous biomarkers of OATP activity. In contrast, the relatively low inter- and intraindividual variability in basal plasma CPs I and III levels makes them especially attractive (Lai et al., 2016). Since CP III is actively secreted in the urine in monkeys and humans (Lai et al., 2016; Shen et al., 2016), we further examined involvement of renal transporter(s) in CP elimination and showed that CPs I and III are not substrates for known major renal drug transporters, including OAT1-4, OCT2, MATE1, and MATE2K (Figs. 5 and 6). In addition, the appropriateness of CPs I and III as markers of OATP activity is supported by lack of substrate-dependent inhibition of CP and RSV transport. OATP1B1 and OATP1B3 displayed comparable affinity for CP I, CP III, and RSV (Figs. 5-7; Table 2) (Shen et al., 2013; Prueksaritanont et al., 2014; Chu et al., 2015; Izumi et al., 2015). Moreover, the $\mathrm{IC}_{50}$ values of $\mathrm{RIF}$ for the inhibition of OATP2B1-mediated CP III and RSV transport differed by less than 2-fold, although RIF was only a weak inhibitor of OATP2B1 (Shen et al., 2013; Prueksaritanont et al., 2014).

In conclusion, following a rigorous comparative analysis, we established that CPs I and III in plasma are the most sensitive biomarkers reflecting OATP inhibition and provided the largest effect size in response to RIF treatment. In addition, we have shown that HDA and TDA, but not BAs and DHEAS, can also serve as sensitive endogenous biomarkers of OATP activity. Incorporating biomarker measurement into drug pharmacokinetics analysis during early drug development, either using one of those biomarkers alone or as a panel, should help avoid expensive clinical trials and lessen the risk of late stage failures owing to severe DDIs. Further studies are needed to understand the differential responses of these biomarkers to various inhibitors beyond RIF and the effects of polymorphisms in OATP proteins and various disease states, including hepatic insufficiency. Additionally, although each of the markers appears to provide a robust response to OATP inhibition, a panel of endogenous compounds that include CP I, CP III, HDA, and TDA may be the method of choice to ensure detection of changes in OATP activity, as individual markers may have differential sensitivities to such other covariates as intestinal and renal transporters and enzyme activities. The findings from the present and previous studies could form the basis for clinical screening of a drug candidate's OATP inhibition potential and may help avoid dedicated druginteraction studies with a probe drug.

\section{Acknowledgments}

The authors thank Dr. Petia Shipkova for guidance in the conduct of the CP and BA analyses.

\section{Authorship Contributions}

Participated in research design: Shen, Mandlekar, Holenarsipur, Gan, Humphreys, Marathe, Lai.

Conducted experiments: Chen, Shields, Langish.

Contributed new reagents or analytic tools: Shen, Chen, Drexler, Shields, Langish, Lai.
Performed data analysis: Shen, Chen, Drexler, Shields, Langish, Sidik, Lai. Wrote or contributed to the writing of the manuscript: Shen, Chen, Drexler, Humphreys, Marathe, Lai.

\section{References}

Alrefai WA and Gill RK (2007) Bile acid transporters: structure, function, regulation and pathophysiological implications. Pharm Res 24:1803-1823.

Bednarczyk D and Boiselle C (2016) Organic anion transporting polypeptide (OATP)-mediated transport of coproporphyrins I and III. Xenobiotica 46:457-466.

Briz O, Romero MR, Martinez-Becerra P, Macias RI, Perez MJ, Jimenez F, San Martin FG, and Marin JJ (2006) OATP8/1B3-mediated cotransport of bile acids and glutathione: an export pathway for organic anions from hepatocytes? J Biol Chem 281:30326-30335.

Chu X, Shih SJ, Shaw R, Hentze H, Chan GH, Owens K, Wang S, Cai X, Newton D, Castro-Perez $\mathrm{J}$, et al. (2015) Evaluation of cynomolgus monkeys for the identification of endogenous biomarkers for hepatic transporter inhibition and as a translatable model to predict pharmacokinetic interactions with statins in humans. Drug Metab Dispos 43:851-863.

Cui Y, König J, Leier I, Buchholz U, and Keppler D (2001) Hepatic uptake of bilirubin and its conjugates by the human organic anion transporter SLC21A6. J Biol Chem 276:9626-9630.

Dawson PA, Lan T, and Rao A (2009) Bile acid transporters. J Lipid Res 50:2340-2357.

Engelking LR, Dasher CA, and Hirschowitz BI (1980) Within-day fluctuations in serum bile-acid concentrations among normal control subjects and patients with hepatic disease. Am J Clin Pathol 73:196-201.

Everson GT (1987) Steady-state kinetics of serum bile acids in healthy human subjects: single and dual isotope techniques using stable isotopes and mass spectrometry. J Lipid Res 28:238-252. Fausa O (1976) Serum bile acid concentration after a test meal. Scand J Gastroenterol 11:229-232. Izumi S, Nozaki Y, Komori T, Maeda K, Takenaka O, Kusano K, Yoshimura T, Kusuhara H, and Sugiyama Y (2013) Substrate-dependent inhibition of organic anion transporting polypeptide 1B1: comparative analysis with prototypical probe substrates estradiol-17 $\beta$-glucuronide, estrone-3-sulfate, and sulfobromophthalein. Drug Metab Dispos 41:1859-1866.

Izumi S, Nozaki Y, Maeda K, Komori T, Takenaka O, Kusuhara H, and Sugiyama Y (2015) Investigation of the impact of substrate selection on in vitro organic anion transporting polypeptide 1B1 inhibition profiles for the prediction of drug-drug interactions. Drug Metab Dispos 43:235-247.

Klaassen CD and Aleksunes LM (2010) Xenobiotic, bile acid, and cholesterol transporters: function and regulation. Pharmacol Rev 62:1-96.

Koenen A, Köck K, Keiser M, Siegmund W, Kroemer HK, and Grube M (2012) Steroid hormones specifically modify the activity of organic anion transporting polypeptides. Eur J Pharm Sci 47: 774-780.

Kullak-Ublick GA, Ismair MG, Stieger B, Landmann L, Huber R, Pizzagalli F, Fattinger K, Meier PJ, and Hagenbuch B (2001) Organic anion-transporting polypeptide B (OATP-B) and its functional comparison with three other OATPs of human liver Gastroenterology 120:525-533.

Kullak-Ublick GA, Stieger B, and Meier PJ (2004) Enterohepatic bile salt transporters in normal physiology and liver disease. Gastroenterology 126:322-342.

Labrie F, Luu-The V, Bélanger A, Lin SX, Simard J, Pelletier G, and Labrie C (2005) Is dehydroepiandrosterone a hormone? J Endocrinol 187:169-196.

Lai Y, Mandlekar S, Shen H, Holenarsipur VK, Langish R, Rajanna P, Murugesan S, Gaud N, Selvam S, Date O, et al. (2016) Coproporphyrins in plasma and urine can be appropriate clinical biomarkers to recapitulate drug-drug interactions mediated by organic anion transporting polypeptide inhibition. J Pharmacol Exp Ther 358:397-404.

Leung A, Schaefer EW, Tempelhof MW, and Stone NJ (2012) Emphasizing statin safety in the hospitalized patient: a review. Am J Med 125:845-853.

Maeda K, Kambara M, Tian Y, Hofmann AF, and Sugiyama Y (2006) Uptake of ursodeoxycholate and its conjugates by human hepatocytes: role of $\mathrm{Na}(+)$-taurocholate cotransporting polypeptide (NTCP), organic anion transporting polypeptide (OATP) 1B1 (OATP-C), and oatp1B3 (OATP8). Mol Pharm 3:70-77.

Marin JJ, Macias RI, Briz O, Banales JM, and Monte MJ (2015) Bile acids in physiology, pathology and pharmacology. Curr Drug Metab 17:4-29.

Meier PJ and Stieger B (2002) Bile salt transporters. Annu Rev Physiol 64:635-661.

Neyt S, Vliegen M, Verreet B, De Lombaerde S, Braeckman K, Vanhove C, Huisman MT, Dumolyn C, Kersemans K, Hulpia F, et al. (2016) Synthesis, in vitro and in vivo small-animal SPECT evaluation of novel technetium labeled bile acid analogues to study (altered) hepatic transporter function. Nucl Med Biol 43:642-649.

Ponz De Leon M, Murphy GM, and Dowling RH (1978) Physiological factors influencing serum bile acid levels. Gut 19:32-39.

Prueksaritanont T, Chu X, Evers R, Klopfer SO, Caro L, Kothare PA, Dempsey C, Rasmussen S, Houle R, Chan G, et al. (2014) Pitavastatin is a more sensitive and selective organic aniontransporting polypeptide 1B clinical probe than rosuvastatin. Br J Clin Pharmacol 78:587-598.

Shen H, Dai J, Liu T, Cheng Y, Chen W, Freeden C, Zhang Y, Humphreys WG, Marathe P, and Lai Y (2016) Coproporphyrins I and III as functional markers of OATP1B activity: in vitro and in vivo evaluation in preclinical species. J Pharmacol Exp Ther 357:382-393.

Shen H, Liu T, Morse BL, Zhao Y, Zhang Y, Qiu X, Chen C, Lewin AC, Wang XT, Liu G, et al (2015) Characterization of organic anion transporter 2 (SLC22A7): a highly efficient transporter for creatinine and species-dependent renal tubular expression. Drug Metab Dispos 43:984-993

Shen H, Yang Z, Mintier G, Han YH, Chen C, Balimane P, Jemal M, Zhao W, Zhang R, Kallipatti $\mathrm{S}$, et al. (2013) Cynomolgus monkey as a potential model to assess drug interactions involving hepatic organic anion transporting polypeptides: in vitro, in vivo, and in vitro-to-in vivo extrapolation. J Pharmacol Exp Ther 344:673-685.

Shimada H, Nakamura Y, Nakanishi T, and Tamai I (2015a) OATP2A1/SLCO2A1-mediated prostaglandin E2 loading into intracellular acidic compartments of macrophages contributes to exocytotic secretion. Biochem Pharmacol 98:629-638.

Shimada T, Nakanishi T, Tajima H, Yamazaki M, Yokono R, Takabayashi M, Shimada T, Sawamoto K, Miyamoto K, Kitagawa H, et al. (2015b) Saturable hepatic extraction of gemcitabine involves biphasic uptake mediated by nucleoside transporters equilibrative nucleoside transporter 1 and 2. J Pharm Sci 104:3162-3169.

Takahashi K, Yoshisue K, Chiba M, Nakanishi T, and Tamai I (2015) Involvement of concentrative nucleoside transporter 1 in intestinal absorption of trifluridine using human small intestinal epithelial cells. J Pharm Sci 104:3146-3153. 
Thakare R, Gao H, Kosa RE, Bi YA, Varma MV, Cerny M, Sharma R, Kuhn M, Huang B, Liu Y, et al. (2017) Leveraging of rifampicin-dosed cynomolgus monkeys to identify bile acid 3-Osulfate conjugates as potential novel biomarkers for organic anion-transporting polypeptides. Drug Metab Dispos DOI: https://doi.org/10.1124/dmd.117.075275.

Tonsgard JH (1986) Serum dicarboxylic acids in patients with Reye syndrome. J Pediatr 109: $440-445$.

Tonsgard JH, Mendelson SA, and Meredith SC (1988) Binding of straight-chain saturated dicarboxylic acids to albumin. $J$ Clin Invest 82:1567-1573.

Watanabe M, Watanabe T, Yabuki M, and Tamai I (2015) Dehydroepiandrosterone sulfate, a useful endogenous probe for evaluation of drug-drug interaction on hepatic organic anion transporting polypeptide (OATP) in cynomolgus monkeys. Drug Metab Pharmacokinet 30:198-204.

Yamaguchi H, Okada M, Akitaya S, Ohara H, Mikkaichi T, Ishikawa H, Sato M, Matsuura M, Saga T, Unno M, et al. (2006) Transport of fluorescent chenodeoxycholic acid via the human organic anion transporters OATP1B1 and OATP1B3. J Lipid Res 47:1196-1202.
Yee SW, Giacomini MM, Hsueh CH, Weitz D, Liang X, Goswami S, Kinchen JM, Coelho A, Zur AA, Mertsch K, et al. (2016) Metabolomic and genome-wide association studies reveal potential endogenous biomarkers for OATP1B1. Clin Pharmacol Ther 100:524-536.

Zheng JJ, Shields EE, Snow KJ, Nelson DM, Olah TV, Reily MD, Robertson DG, Shipkova PA, Stryker SA, Xin B, et al. (2016) The utility of stable isotope labeled (SIL) analogues in the bioanalysis of endogenous compounds by LC-MS applied to the study of bile acids in a metabolomics assay. Anal Biochem 503:71-78.

Address correspondence to: Dr. Hong Shen, Bristol-Myers Squibb Company, Route 206 and Province Line Road, Princeton, NJ 08543-4000. E-mail: hong. shen1@bms.com 